\title{
Diagnostic delays in rheumatic diseases with associated arthritis
}

\author{
Filip Raciborski ${ }^{1}$, Anna Kłak², Brygida Kwiatkowska ${ }^{3}$, Bogdan Batko4, \\ Małgorzata Sochocka-Bykowska ${ }^{5}$, Aleksandra Zoń-Giebel ${ }^{6}$, Zbigniew Gola ${ }^{7}$, Zbigniew Guzera ${ }^{8}$, \\ Maria Maślińska ${ }^{3}$, working team*
}

* J. Grygielska, M. Mańczak, S. Ostrowska, P. Samel-Kowalik

\begin{abstract}
${ }^{1}$ Department of Prevention of Environmental Hazards and Allergology, Medical University of Warsaw, Poland ${ }^{2}$ Department of Gerontology, Public Health and Didactics, National Institute of Geriatrics, Rheumatology and Rehabilitation, Warsaw, Poland ${ }^{3}$ Clinic of Early Arthritis, National Institute of Geriatrics, Rheumatology and Rehabilitation, Warsaw, Poland ${ }^{4}$ Department of Rheumatology, J. Dietl Specialist Hospital, Krakow, Poland ${ }^{5}$ Vivodal Rheumatology Hospital, Sopot, Poland ${ }^{6}$ Silesian Centre of Rheumatology, Rehabilitation and Desability Prevention Gen J. Ziętka, Ustroń, Poland ${ }^{7}$ SENSUS Speciality Hospital LLC, Stalowa Wola, Poland

${ }^{8}$ Department of Rheumatology District Healtcare Unit of Starachowice, Poland
\end{abstract}

\begin{abstract}
Objective: The objective of this study was to determine the length of delay in diagnosis of inflammatory rheumatic diseases, and to indicate the main factors responsible for such delays.

Material and methods: A retrospective multi-centre questionnaire survey carried out among 197 patients with diagnosed inflammatory rheumatic diseases or undergoing the diagnostic process. Results: The most common early symptoms of inflammatory rheumatic disease included joint pain (94\%), joint swelling (78\%), morning joint stiffness (77\%), fatigue (76\%), and sleep disturbed by joint pain (74\%). When asked about the reasons for seeking medical help, most patients indicated intensification of the symptoms (89\%) and the fact that the symptoms made them unable to perform daily activities or work (86\%). Limited access to specialists (70\%) and the conviction that the symptoms will resolve spontaneously (57\%) had the biggest impact on delaying a visit to a doctor. Before visiting a rheumatologist, the patients consulted their symptoms with their general practitioners (GPS, 95\%), orthopaedicians (43\%), and neurologists (29\%). Almost half of the patients (48\%) consulted their symptoms with at least 2 non-rheumatologists, whereas as many as $21 \%$ of patients visited 4 or more specialists. After the onset of symptoms of rheumatic disease, $28 \%$ of patients delayed seeing any doctor for 4 months or longer. $36 \%$ of patients waited 4 months or longer for a referral to a rheumatologist. The great majority of the patients (85\%) made an appointment with a rheumatologist within a month of receiving a referral. $25 \%$ of patients waited 4 months or longer to see a rheumatologist.

Conclusions: Diagnostic delays result from both the level of patients' awareness (ignoring early symptoms) and improper functioning of the health care system. In the case of the health care system, the source of delays is not only "queues to rheumatologists", but also referring patients to non-rheumatologists.
\end{abstract}

Key words: inflammatory rheumatic diseases, diagnostic delays, risk factors. 


\section{Introduction}

Early diagnosis of inflammatory joint and connective tissue disease is of key importance in achieving effective treatment and improved prognosis. It is also indirectly reflected in a patient's quality of life. Early joint inflammation requires strict monitoring and verification of such diagnosis to exclude early rheumatoid arthritis (RA) or early non-radiographic ${ }^{1}$ spondyloarthropathy as quickly as possible - before the development of radiographic changes and other inflammatory rheumatic diseases. It matters because the strategy of treatment of these disease entities is different. Inflammatory rheumatic disease should be diagnosed as soon as possible, and effective treatment should be started immediately after diagnosis is made. In its early period, this disease requires intense monitoring with regard to effectiveness and safety of treatment in order to achieve remission in the shortest time possible [1].

According to the European League Against Rheumatism (EULAR) recommendations from 2016, each patient with persistent swelling in at least one joint should be referred to a rheumatologist and examined within 6 weeks of the onset of symptoms, and effective treatment should be started by the end of the $12^{\text {th }}$ week [2]. Due to the key importance of early diagnosis of inflammatory joint disease, the so-called therapeutic window of opportunity was introduced, which in the case of RA is 12 weeks from the onset of symptoms to diagnosis and commencement of effective treatment [2]. It is of vital importance to treat every single symptom of joint inflammation, rather than the appearance of joint swelling, as early symptoms of this disease [3]. The term "therapeutic window of opportunity" was first introduced for rheumatoid arthritis. The analysis of negative consequences of the time from symptoms onset to diagnosis and commencement of effective treatment in other inflammatory diseases (such as spondyloarthropathies and systemic lupus erythematosus) allowed implementation of the term "therapeutic window of opportunity" also in these disease entities. In contrast to RA, the time interval is not defined here. This is due to the fact that "diagnostic delays" in these diseases are currently a couple of months, or even years [4].

In recent years it has been shown that the only predictive factor to achieve remission in RA is early diagnosis and immediate treatment [5-7]. Starting treatment within 12 weeks of symptom onset doubles the chance of achieving remission, and the necessity to use biological medicinal products in RA treatment decreases from $32.2 \%$ to $10 \%$ [8]. Therefore, new diagnostic criteria for
RA have been developed [9]. They do not include the changes - usually late ones - in radiographic examinations. In addition, studies are being carried out to determine factors that can help diagnose this disease before the onset of typical clinical symptoms. This will enable early intervention aimed at delaying the disease progression. According to EULAR guidelines published in 2017, risk factors for development of RA in patients with arthralgia include: joint pain lasting less than a year, symptoms appearing in metacarpophalangeal joints, morning joint stiffness lasting $\geq 60 \mathrm{~min}$, intensification of symptoms in the morning hours, and during physical examination - difficulties clenching one's fist and a positive squeeze test of MCP joints [9]. The aforementioned characteristics concern patients with joint pain, but without clinical inflammation. European studies have shown that the total cost of diagnosis and treatment of one patient with early diagnosed joint inflammation is 2424 Euro [10]. In the case of late diagnosis, this cost is twice as high - 5928 Euro [10], which proves that investing money in early diagnostics of joint inflammation is economically justified as far as the payer is concerned, and it allows a reduction future treatment costs of advanced disease.

The objective of this study was to determine the length of delay in diagnosis of inflammatory rheumatic diseases, and to indicate the main factors responsible for such delays.

\section{Material and methods}

The quantitative study was based on a questionnaire developed by the researchers, and completed individually by each patient. This tool consisted of 18 basic and 5 socio-demographic questions. Some questions had a multiple-choice format. The questionnaire included questions regarding early symptoms of disease, their after-effects, and patients' help-seeking paths.

The following institutions were responsible for the execution of this multi-centre study: National Institute of Geriatrics, Rheumatology and Rehabilitation (Warsaw), Non-Public Health Care Centre Outpatient Clinic for Osteoporosis and Locomotive System Diseases (Stalowa Wola), Silesian Centre for Rheumatology, Rehabilitation and Disability Prevention (Ustroń), Dietl Specialist Hospital in Krakow, and Provincial Rheumatology Clinic in Sopot. The study was conducted among both hospitalised patients and patients using outpatient specialist care. Two groups of patients were qualified to the study: 1) with diagnosed inflammatory rheumatic disease (diagnosed within 12 months before the study), and 2) un-

${ }^{1}$ Inflammatory changes are not yet visible in a standard X-ray picture. 
dergoing diagnosis. Only those patients with a definitive diagnosis of arthritis were included, and there were no reasons for referral, so the accuracy of the initial diagnosis of the referral physician could not be assessed (the relevance of the analysis at the screening stage). The selection of patients to the study was based on the degree of cooperation. The exclusion criterion was age below 18 years. Due to the fact that it was not a representative study, and its results should not be extrapolated to an entire population, confidence intervals were not given. The study had a descriptive, not analytical, character. The results were presented as the frequency of replies to particular questions. The characteristics of the study group are presented in Table I.

The study was conducted between May 2014 and May 2015. A total of 500 questionnaires with instructions for patients and doctors responsible for the execution of the study were given to the centres. Out of 239 received questionnaires, 234 were accepted (fully completed questionnaires). 197 questionnaires, either from patients undergoing diagnosis or from those with diagnosed inflammatory rheumatic disease, were included in the final analysis because people who reported osteoarthritis or osteoporosis as the only rheumatic disease were excluded from the study. Patients who reported osteoarthritis or osteoporosis and at least one other inflammatory disease were included in the study.

In the case of multiple choice questions, some respondents marked only affirmative replies. In such situations, non-marked categories were interpreted as negative replies.

The study obtained the approval of the Bioethics Committee at the National Institute of Geriatrics, Rheumatology and Rehabilitation (approvals from 31 May 2012 and 27 November 2014).

\section{Results}

The most common early symptom of rheumatic disease that appeared before visiting any specialist was joint pain, declared by $94 \%$ of respondents. In the group of people without joint pain (6\%), there were other symptoms of rheumatic diseases that were the cause of diagnosis for inflammatory diseases (e.g swelling). Nearly four out of every five patients (78\%) recorded joint swelling. A similar percentage of the people reported morning joint stiffness (77\%), and fatigue (76\%). Frequent early symptoms included also sleep disturbed by pain (74\%), as well as tingling and numbness in the hands and legs (67\%). The detailed data are presented in Figure 1.

The patients participating in the study were also asked to define the inconvenience arising from their
Table I. Characteristics of the study group

\begin{tabular}{|c|c|}
\hline Gender $(n=197)$ & $\%$ \\
\hline Women & 77 \\
\hline Men & 23 \\
\hline Age $(n=196)$ median (min.-max.) & $\begin{array}{c}50.5 \\
\text { years } \\
(18-86)\end{array}$ \\
\hline 18-34 years & 20 \\
\hline $35-49$ years & 27 \\
\hline 50-64 years & 37 \\
\hline 65 years and older & 16 \\
\hline \multicolumn{2}{|l|}{ Status on the labour market $(n=192)^{*}$} \\
\hline Working & 55 \\
\hline Pensioner & 30 \\
\hline Student & 7 \\
\hline Unemployed & 7 \\
\hline Unemployed (another reason) & 5 \\
\hline Volunteer & 1 \\
\hline \multicolumn{2}{|l|}{ Geographic residence $(n=192)$} \\
\hline Village & 33 \\
\hline City & 67 \\
\hline \multicolumn{2}{|l|}{ Diagnosis $(n=197)^{*}$} \\
\hline Disease has not been diagnosed yet & 51 \\
\hline Rheumatoid arthritis (RA) & 31 \\
\hline Osteoarthritis & 11 \\
\hline Osteoporosis & 8 \\
\hline Ankylosing spondylitis (AS) & 7 \\
\hline Psoriatic arthritis (PA) & 6 \\
\hline Systemic lupus erythematosus & 2 \\
\hline Juvenile idiopathic arthritis (JIA) & 1 \\
\hline Other & 9 \\
\hline \multicolumn{2}{|l|}{ Place of the study $(n=197)$} \\
\hline Krakow & 14 \\
\hline Sopot & 13 \\
\hline Stalowa Wola & 9 \\
\hline Starachowice & 15 \\
\hline Ustroń & 21 \\
\hline Warsaw & 28 \\
\hline
\end{tabular}

* Multiple choice question. The results do not sum up to 100\%

symptoms. On the scale of $1-5$ (where 5 means very severe inconvenience), joint pain had a mean value of 3.9 points, whereas morning joint stiffness -3.8 points (only the people with a given symptom were included in the analysis). In the case of sleep disturbed by joint pain and joint swelling, the degree of inconvenience oscillated at 


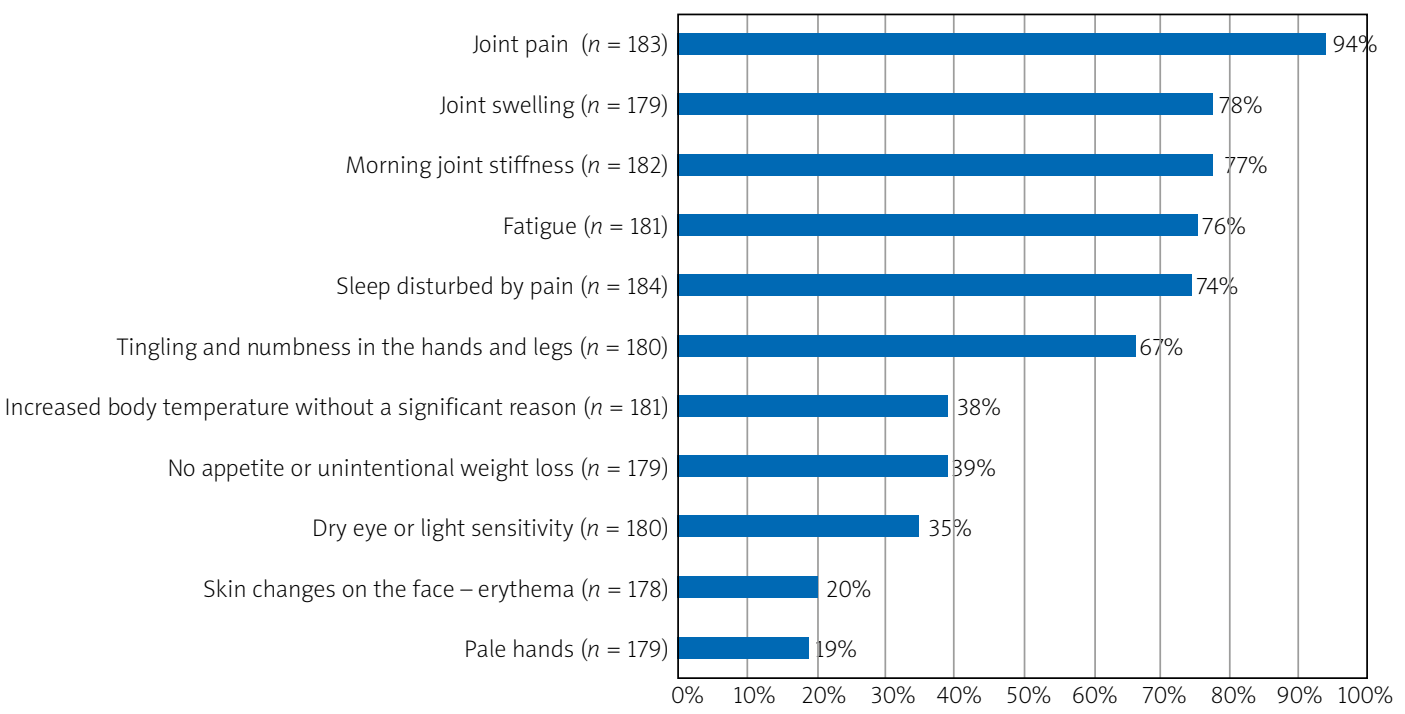

Fig. 1. The percentage of people declaring the presence of given symptoms before seeing a doctor.

3.6-3.7 points. Fatigue received 3.4 points, and tingling and dumbness -3.2 points.

Over two-third of the respondents (69\%) used (overthe-counter) painkillers and anti-inflammatory medications to relieve the symptoms before consulting them with a doctor. $65 \%$ of patients used various types of compresses, gels, and ointments. Every second respondent (51\%) used prescription painkillers and anti-inflammatory medications. Over one-third of the respondents (37\%) declared using rehabilitation, physical therapy, or massages to relieve the symptoms. Over one-fifth of the respondents (22\%) used herbal preparations. Every eighth person (12\%) used alternative medicine. Only $7 \%$ of patients reported they had not used any methods to relieve the symptoms connected with rheumatic diseases before visiting a doctor.

When asked about the reasons for seeking medical help, most patients indicated exacerbation of the symptoms (89\%) and the fact that the symptoms made them unable to perform daily activities or work (86\%). Limited access to specialists (70\%) had the biggest impact on delaying a visit to a doctor. Nearly three out of every five respondents (57\%) indicated that delaying a visit to a doctor was influenced by the conviction that the symptoms would resolve spontaneously. The detailed data are presented in Figures 2A and 2B.

Before seeing a rheumatologist, the patients most often consulted the symptoms of rheumatic diseases with their GPs (95\%), orthopaedicians (43\%), and neurologists (29\%). The detailed data are presented in Figure 3. Before visiting an orthopaedician, one out of every three patients (34\%) consulted the symptoms of rheumatic diseases with only one doctor. Twenty-eight percent of respondents consulted two different specialists, whereas $18 \%$ - three specialists. About $21 \%$ visited four or more specialists (before seeing a rheumatologist).

Two-third of the respondents (66\%) were referred to a rheumatologist by their GPs. Every ninth patient (11\%) was referred to an orthopaedician. About $21 \%$ of respondents reported other specialists. Five respondents (3\%) did not use a referral to a rheumatologist (they probably used non-public health care services).

Diagnostic delays were analysed in four different categories:

- the time from onset of symptoms to seeing any specialist,

- the time from the first visit to a doctor to receiving a referral to a rheumatologist,

- the time from receiving a referral to making an appointment with a rheumatologist,

- the time from making an appointment to visiting a rheumatologist.

In $50 \%$ of respondents, the time from onset of symptoms to the first visit to a doctor was less than one month. $22 \%$ of patients consulted their symptoms within 1 to 3 months of their onset. $28 \%$ of respondents delayed a visit for four months or longer.

Among $40 \%$ of respondents, the time from the first visit to a doctor to receiving a referral to a rheumatologist was nearly one month. Twenty-four percent of the respondents had to wait from 1 to 3 months for a referral. $36 \%$ of patients were referred to a rheumatologist after 4 months or later.

Over half of the patients (56\%) made an appointment with a rheumatologist immediately after receiving a referral (i.e. within 7 days). Within one month $-85 \%$, within 3 months - 92\%. 

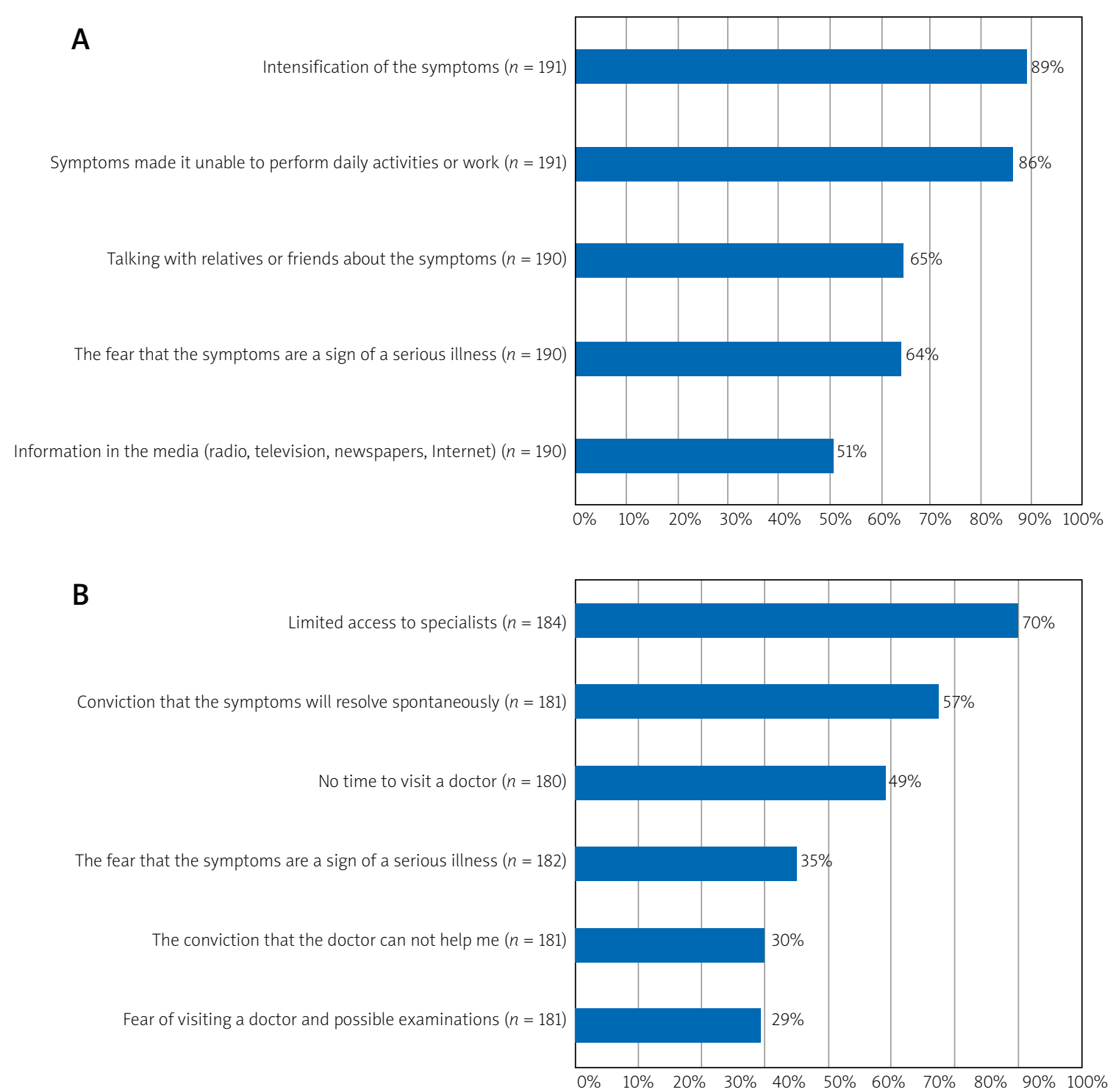

Fig. 2. The percentage of people declaring the impact of a given factor on seeking medical help (A). The percentage of people declaring the impact of a given factor on delaying a visit to a doctor (B).

Forty-six percent of respondents had their first visit to a rheumatologist within one month of making an appointment. In $29 \%$ of patients this time was from 1 to 3 months, and in 25\% - 4 months or longer. The detailed data are presented in Figure 4.

\section{Discussion}

This study was conducted using a questionnaire, on the basis of patients' declarations. Due to a retrospective character of the study, there was a risk that the information given by the respondents might not be exact.
Therefore, the authors of the study took into consideration only patients who had been diagnosed within the last 12 months preceding the study, or were undergoing diagnosis. This allowed limiting the influence of time on the quality of the respondents' information.

The patients were enrolled in this multi-centre study on the basis of inclusion criteria. The sampling method did not guarantee the representativeness of the study. The authors were not able to evaluate to what extent the characteristics of the study group were compliant with the characteristics of the target population (people with inflammatory joint diseases diagnosed with- 


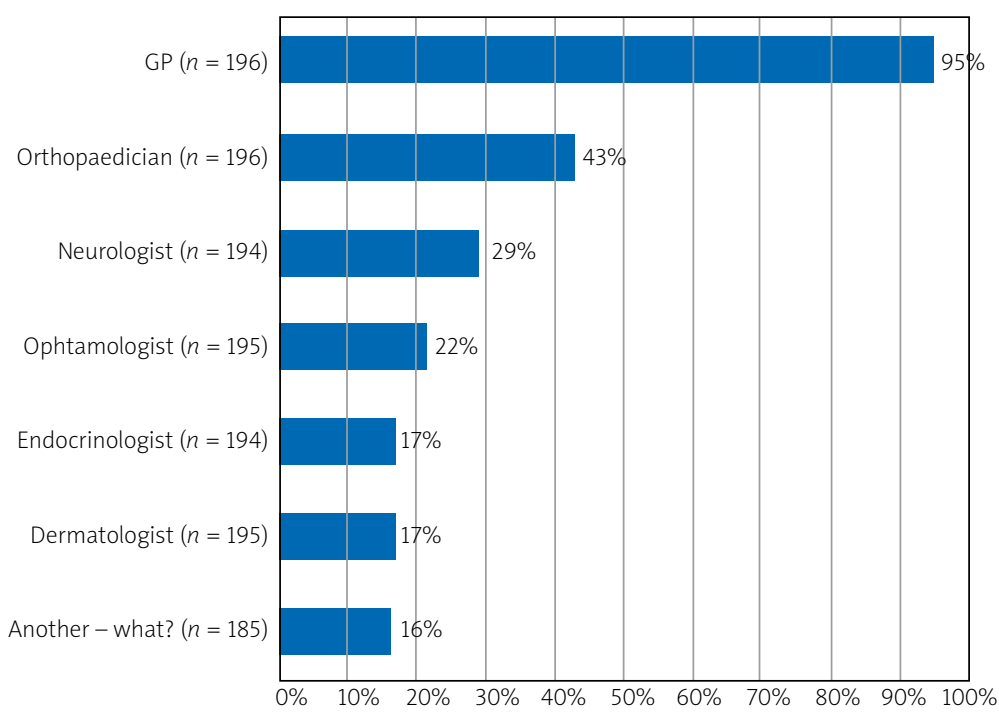

Fig. 3. Doctors visited by patients with the symptoms of rheumatic disease, before the first visit to a rheumatologist.

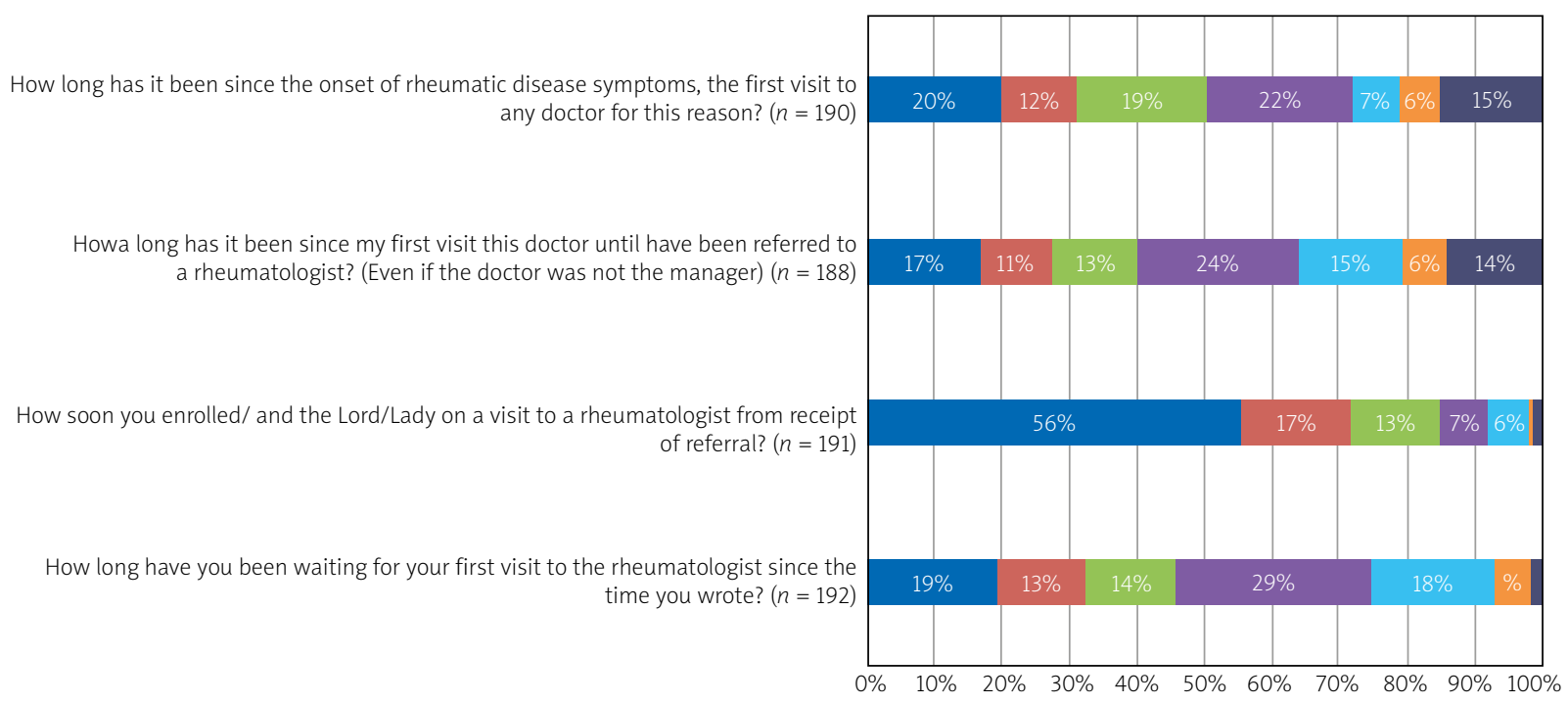

Fig. 4. Diagnostic delays in rheumatic diseases depending on the stage of diagnosis/treatment.

in the last 12 months, or undergoing diagnosis). No comparative data are available. Also no analysis was made regarding the respondent's place of residence (village/city). The authors did not analyse the distance the patient had between the hospital and the place of residence. The aforementioned methodological limitations, however, concern most studies - both single- and multi-centre ones.

Over half (51\%) of the people in the study group were undergoing diagnosis. According to the assumptions, the study focused on inflammatory rheumatic diseases. In some of the people undergoing diagnosis, the symp- toms might indicate non-inflammatory disease. Nevertheless, excluding the group of the people undergoing diagnosis from the study would have a negative impact on the substantive value of the project. The responses of the people undergoing diagnosis regarding, among others, early symptoms, previous medical consultations, or diagnostic delays are least susceptible to the passage of time and the forgetting effect.

The respondents' replies regarding use of prescription anti-inflammatory medications and painkillers before the first visit to a doctor are interesting. It can be assumed that the respondents either did not under- 
stand this question, or used medications they already had at home (e.g. prescribed during previous therapy or belonging to another member of the family).

In this study, diagnostic delays dependent on a patient included delays connected with scheduling the first appointment with a physician, and that of making an appointment with a rheumatologist after receiving a referral. In the case of the former category, as many as $28 \%$ of respondents waited 4 months or longer. In the case of RA, it would mean that a patient starts using the services of the health care system after the so-called therapeutic window of opportunity, which is 12 weeks for this disease entity. The results of the study comply with the results of many other international studies that indicate that increase and intensification of the symptoms have the greatest impact on diagnostic delays in $\operatorname{RA}[6,11]$. There is a lack of social awareness as far as the symptoms of rheumatic diseases and the importance of a quick diagnosis are concerned $[6,12,13]$.

The results obtained in this study differ from the results obtained for the inhabitants of Warsaw by Raza's team in 2010. They showed that the mean time from onset of the symptoms of RA to a visit to a rheumatologist was 35 weeks [14]. This is much longer than European recommendations assume, which is of key importance for the future of patients in the Polish health care system. The median wait time from onset of the symptoms of RA to the first visit to a rheumatologist was 24 weeks for all 10 European centres taking part in the Raza study [14]. To compare, in Holland this period was nearly 14 weeks [15].

Early diagnosis of spondyloarthropathy and RA has a significant impact on the effectiveness of treatment and the chance of remission. Some studies show that diagnostic delays in spondyloarthropathy are even greater than in the case of RA. Feldtkeller et al. [4] showed that in $59 \%$ of patients with spondyloarthropathy the time from onset of symptoms to the diagnosis was more than 10 years. Shortening the time from onset of symptoms to the diagnosis is possible due to new ASAS (Assessment of SpondyloArthritis 2010) criteria for diagnosing axial and peripheral spondyloarthropathies.

Early diagnosis and the commencement of effective treatment are also important in the case of systemic lupus erythematosus. In this disease, the time from onset of symptoms to the diagnosis is often over 30 months [16]. This is the case particularly in patients whose early symptoms include joint pain and/or swelling [16].

According to NICE Quality Standard, people with persistent synovitis of small joints of the hands or feet, or of more than one joint, should consult a rheumatologist within 3 days of the first visit to a primary care doctor [17]. Nonetheless, available analyses indicate that the time between using primary health care and visiting a rheumatologist has the greatest impact on a diagnostic delay [14]. Our study has showed that $40 \%$ of patients received a referral to a rheumatologist within one month of the first visit to a primary care doctor. Every fourth patient had to wait from one to three months for a referral, and every third patient - even 4 months or longer. The results of the national audit conducted in Great Britain show, in turn, that in the time interval recommended by NICE, $17 \%$ of patients visit a rheumatologist (it should be noted that in Great Britain patients with this disease wait on average 34 days for a visit to a rheumatologist, and only $25 \%$ of patients wait longer than 3 months) [17].

The NICE guidelines also state that people with suspected persistent synovitis should be diagnosed by a rheumatologist within 3 weeks of referral. In Great Britain, 38\% of patients meet the NICE criteria, and the median wait time for a visit to a rheumatologist in this case is 4 weeks [17]. The result obtained in our study, showing that nearly every second respondent had the first visit to a rheumatologist within one month of making an appointment with this specialist, can be considered satisfactory. At the same time, it should be noted that every third patient waited from one to three months for a visit to a rheumatologist, and every fourth respondent -4 months or longer.

Data published by the National Health Fund [Narodowy Fundusz Zdrowia, NFZ] (as on 31 May 2014) show that the median wait time for rheumatology ambulatory care for stable patients in Poland is 28 days (median) [18]. Out of 727 entities registered in Poland, 141 declared that admissions took place on a current basis. However, for nearly $9 \%$ of entities the wait time was over 90 days. There are also facilities where patients have to wait over 400 days for a visit to a rheumatologist. According to the data from the National Health Fund, urgent cases are admitted on a current basis in $80 \%$ of facilities. In about $10 \%$ of facilities the wait time for urgent cases is 10 days or longer. In the Lower Silesian and Opole voivodeships, there are outpatient clinics where the declared wait time for a visit in the case of urgent cases is 80 days or longer [18].

It should be noted, however, that the credibility of the data from the National Health Fund can be limited as they are based on declarations of particular medical entities. The study conducted for the needs of the report "ME, PATIENT! The View of Patient Organisations on the State of Rheumatology Care in Poland" shows that the real wait time for rheumatology services can be nearly twice longer than the one declared by the National Health Fund [19]. This issue requires further analyses. In the case of inflammatory joint diseases, diagnosis and 
commencement of treatment during the therapeutic window of opportunity unequivocally determines patients' chances for remission. Unfortunately, it is currently not reflected in the rheumatology care system in Poland. Poland is a country where the time from diagnosis to commencement of treatment is one of the longest in Europe [14], which significantly reduces the effectiveness of treatment, increases its costs, and contributes to the increase in pension benefits and sickness absenteeism [18].

\section{Conclusions}

In the first period from onset of the symptoms of rheumatic diseases (before visiting a doctor) most people use self-treatment. Taking anti-inflammatory medications and painkillers that reduce the symptoms can lead to delaying the decision about medical consultation.

Only $34 \%$ of patients who seek medical help due to the symptoms of rheumatic diseases are referred directly to a rheumatologist. The remaining people are referred to other specialists, which delays the diagnosis.

Two-thirds of patients receive referrals to rheumatologists from their GPs. Therefore, one should pay special attention to train this group of doctors within rheumatic diseases.

Diagnostic delays depend both on the factors connected directly with the health care system (e.g. wait time for a visit), and with decisions taken by patients (e.g. delaying a visit to a doctor, self-treatment). Actions to limit diagnostic delays should include both organisational changes in health care, and increasing social awareness regarding rheumatic diseases.

The authors declare no conflict of interest.

\section{References}

1. Van Eijk IC, Nielen MMJ, Van der Horst-Bruinsma I, et al. Aggressive therapy in patients with early arthritis results in similar outcome compared with conventional care: the STREAM randomized trial. Rheumatology 2012; 51: 686-694.

2. Smolen JS, Landewé R, Bijlsma J, et al. EULAR recommendations for the management of rheumatoid arthritis with synthetic and biological disease-modifying antirheumatic drugs: 2016 update. Ann Rheum Dis 2017; 76: 960-977.

3. Raza K, Saber TP, Kvien TK, et al. Timing the therapeutic window of opportunity in early rheumatoid arthritis: proposal for definitions of disease duration In clinical trials. Ann Rheum Dis 2012; 71: 1921-1923.

4. Feldtkeller E, Khan MA, van der Heijde D, et al. Age at disease onset and diagnosis delay in HLA-B27 negative vs. positive patients with ankylosing spondylitis. Rheumatol Int 2003; 23: 61-66.

5. van der Linden MP, Cessie S, Raza K, et al. Long-term impact of delay in assessment of patients with early arthritis. Arthritis Rheum 2010; 62: 3537-3546.

6. Stack RJ, Shaw K, Mallen C, et al. Delays in help seeking at the onset of the symptoms of rheumatoid arthritis: a systematic synthesis of qualitative literature. Ann Rheum Dis 2012; 71: 493-497.

7. Atzeni F, Sarzi-Puttini P. Rheumatoid arthritis: Why wait? Explaining delays in seeking therapy for early arthritis. Nat Rev Rheumatol 2012; 8: 190-191.

8. Gremese E, Salaffi F, Bosello SL, et al. Very early rheumatoid arthritis as a predictor of remission: a multicentre real life prospective study. Ann Rheum Dis 2013; 22: 858-862.

9. van Steenbergen HW, Aletaha D, Beaart-van de Voorde LJ, et al. EULAR definition of arthralgia suspicious for progression to rheumatoid arthritis. Ann Rheum Dis 2017; 76: 491-496.

10. Filipon E, Brazier M, Clavel G, et al. Is it possible to identify early predictors of the future cost of chronic arthritis? The VErA project. Fundam Clin Pharmacol 2009; 23: 105-113.

11. Mølbaek K, Hørslev-Petersen K, Primdahl J. Diagnostic Delay in Rheumatoid Arthritis: A Qualitative Study of Symptom Interpretation Before the First Visit to the Doctor. Musculoskeletal Care 2016; 14: 26-36.

12. Barnabe C, Xiong J, Pope JE, et al. Factors associated with time to diagnosis in early rheumatoid arthritis. Rheumatol Int 2014; 34: 85-92.

13. Villeneuve E, Nam JL, Bell MJ, et al. A systematic literature review of strategies promoting early referral and reducing delays in the diagnosis and management of inflammatory arthritis. Ann Rheum Dis 2013; 72: 13-22.

14. Raza K, Stack R, Kumar K, et al. Delays in assessment of patients with rheumatoid arthritis: variations across Europe. Ann Rheum Dis 2011; 70: 1822-1825.

15. van der Linden MP, Cessie S, Raza K, et al. Long-term impact of delay in assessment of patients with early arthritis. Arthritis Rheum 2010; 62: 3537-3546.

16. Ozbek S, Sert M, Paydas S, et al. Delay in the Diagnosis of SLE: The Importance of Arthritis/Arthralgia as the Initial Symptom. Acta Med Okayma 2003; 57: 187-190.

17. Firth J, Snowden N, Ledingham J, et al. The 1st National Clinical audit for Rheumatoid and Early Inflammatory Arthritis: findings and implications for nursing practice. Br J Nurs 2016; 25 : 613-617.

18. Kwiatkowska B, Raciborski F, Maślińska M, et al. Wczesna diagnostyka chorób reumatycznych - ocena obecnej sytuacji i rekomendacje zmian. Instytut Reumatologii w Warszawie, Warszawa 2014.

19. Kotarba-Kańczugowska M, Kucharski K, Linder-Kopiecka I, et al. JA PACJENT! Perspektywa Organizacji Pacjenckich na Stan Opieki Reumatologicznej w Polsce. Raport Organizacji Pacjenckich, Warszawa 2014. 